CERN-TH.7500/94/Revised

astro-ph/9507051

\title{
Experimental limits to the density of dark matter in the solar system
}

\author{
Øyvind Grøn \\ Oslo College, Faculty of Engineering, Cort Adelers Gate 30, N-0254 Oslo, Norway \\ Institute of Physics, University of Oslo, P.O. Box 1048, N-0316 Blindern, Oslo 3, Norway \\ Harald H. Soleng \\ CERN, Theory Division, CH-1211 Geneva 23, Switzerland \\ (16 November 1994; revised 8 June 1995)
}

\begin{abstract}
On the scales of galaxies and beyond there is evidence for unseen dark matter. In this paper we find the experimental limits to the density of dark matter bound in the solar system by studying its effect upon planetary motion.

Subject Headings: Gravitation — Dark Matter (solar system tests)

PACS numbers: 04.20.-q 95.35.+d
\end{abstract}

To appear in the Astrophysical Journal

Typeset using REVTEX 


\section{INTRODUCTION}

According to Newton's inverse square force law, the circular speed around an isolated object of mass $M$ should be

$$
v_{c}=\sqrt{\frac{M G}{r}} .
$$

In disk galaxies we do, however, observe that the circular speeds are approximately independent of $r$ at large distances. The standard explanation is that this is due to halos of unseen matter that makes up around 90\% of the total mass of the galaxies (Tremaine 1992).The same pattern repeats itself on larger and larger scales, until we reach the cosmic scales where a baryonic density compatible with successful big bang nucleosynthesis is less than $10 \%$ of the density predicted by inflation, i.e. the critical density.

The flat rotation curves of galaxies, taken at face value, imply that the effective gravitational force follows a $1 / r$ law at large scales. This could either be due to dark matter or to a departure from Newtonian dynamics at small accelerations (Milgrom 1983; Bekenstein 1992) or large scales (Sanders 1990). An effective gravitational acceleration law of the form

$$
g=-\frac{\sqrt{G M a_{0}}}{r}
$$

at small accelerations $a \ll a_{0}$ has been reported (Kent 1987; Milgrom 1988; Begeman, Broeils, \& Sanders 1991) to be successful in reproducing the observations of galactic systems. The constant $a_{0}$ has been determined by studies of galaxy rotation curves and its value has been found to be $a_{0} \approx 10^{-8} \mathrm{~cm} \mathrm{~s}^{-2}$. As noted by Milgrom (1983), this value of $a_{0} \approx c H_{0}$.

With such a $1 / r$ force law the circular speed would approach $v_{c}=\left(G M a_{0}\right)^{1 / 4}$. If the luminosity $L$ of a galaxy is proportional to its mass $M$, then this relation would explain the infrared Tully-Fisher law (Tully \& Fisher 1977) which states that circular speeds in galaxies scale as $v_{c} \propto L^{1 / 4}$.

The theoretical underpinning for the $1 / r$ effective force law is not yet firmly established. It might be due to a modification of gravity along the lines of Milgrom (1983), but it seems to be difficult to construct a viable relativistic theory of this kind (Zhytnikov and Nester, 1994). Accordingly, the standard view is that the effective galactic $1 / r$ force law is caused by dark matter. At this point, it is worth mentioning that a large-distance force law of this type can be reproduced within standard general relativity theory with a very simple, but perhaps unrealistic, matter source (Soleng 1993, 1995). Our key point is that general relativity is quite capable of explaining the observed gravitational properties of the universe provided we give it the right input. Most likely the dark matter is a mixture of several components, such as weakly interacting particles, black holes, brown dwarfs, neutron stars, as well as energy stored in high-frequency oscillation of Newton's gravitational coupling (Accetta \& Steinhardt 1991; Steinhardt \& Will 1994). Whatever the origin of the $1 / r$ force law might

\footnotetext{
${ }^{1}$ However, not without debate (Lake 1989; Milgrom 1991).
} 
be, its reported experimental success forces us to take it seriously. Accordingly, we think that it is particularly important to compare the densities of dark matter inferred from large scale dynamics with experimental limits from local tests. If dark matter exists in the form of microscopic objects, one would expect that this unknown form of energy penetrates into galaxies and also enter the solar system.

Braginsky, Gurevich, and Zybin (1992) have studied the effect of dark matter bound in the galaxy but unbound to the solar system. Such unbound dark matter would produce an anisotropy in the gravitational background of the solar system. The resulting tidal forces induce an additional perihelion precession. Assuming $\rho_{d}=0.3 \mathrm{GeV} / \mathrm{cm}^{3}=5.4 \times 10^{-25} \mathrm{~g} / \mathrm{cm}^{3}$ they computed the magnitude of the resulting secular orbit distortion. The effect may be observed by reasonable improvements of present observational techniques (Klioner and Soffel 1993; Braginsky 1994). A possible influence of dark matter on the Earth-Moon system has been considered by Nordtvedt (1994) and by Nordtvedt, Müller, and Soffel (1995).

In this paper we focus on a dark matter model in which the density of dark matter varies so slowly within the solar system that it can considered constant. This is a reasonable assumption if dark matter in the solar system really is in the much deeper potential of the galaxy with the Sun causing only a local density perturbation in the galactic dark matter background. It will also be assumed that the equation of state of the unseen matter is almost dust-like, that is, the pressure will be assumed to be much less than the energydensity. Based on this model we calculate an upper limit to the density of dark matter by considering its effect upon the perihelion precession of the planets. We have also carried out similar computations with a dark matter density proportional to $1 / r^{4}$ and $1 / r^{2}$ using the results in Soleng (1994) and (1995), respectively. The corresponding experimental bounds do not vary more than one order of magnitude. This weak dependence on the distribution function corroborates the claim of Anderson et al. (1989) and should be expected because (to lowest order) the perihelion precession caused by dark matter is given by the integrated dark matter mass at a given orbital radius.

\section{SOLAR SYSTEM WITH DARK MATTER}

In order to study the gravitational effects of hypothetical dark matter on planetary motion, we need a solution of Einstein's field equations for a static, spherically symmetric space-time and a given distribution of dark matter. The line-element for a static, spherically symmetric gravitational field can in general be written ast

$$
d s^{2}=-e^{2 \mu(r)} d t^{2}+e^{2 \lambda(r)} d r^{2}+r^{2} d \Omega^{2} .
$$

We shall assume that the dark matter has a constant density $\rho_{0}$ (within the solar system). At a surface where the dark matter pressure equals the galactic dark matter pressure $p_{\mathrm{G}} \approx$ $10^{-7} \rho_{\mathrm{G}}$ (characterized by velocities of $220 \mathrm{~km} / \mathrm{s}$ ), we match the gravitational field of the solar system to the exterior field of the galaxy. We shall assume that $g_{t t} \approx-1$ at this distance

\footnotetext{
${ }^{2}$ We employ geometrized units with $G=c=1$.
} 
(this assumption is always used in local gravitational problems). Then the $t t$-component of the field equations takes the form

$$
\frac{d}{d r}\left[r\left(1-e^{-2 \lambda}\right)\right]=8 \pi \rho_{0} r^{2}
$$

Integrating with a spherical mass $M$ (the Sun) at $r=0$ gives

$$
e^{-2 \lambda}=1-\frac{2 M}{r}-\frac{8 \pi}{3} \rho_{0} r^{2} .
$$

The Tolman-Oppenheimer-Volkov (TOV) equation takes the form

$$
\frac{d p}{d r}=-\left(\rho_{0}+p\right) \frac{M+\frac{4 \pi}{3} \rho_{0} r^{3}+4 \pi p r^{3}}{r^{2}-2 r\left(M+\frac{4 \pi}{3} \rho_{0} r^{3}\right)},
$$

where $p$ is the pressure of the dark matter. Using that the mass of dark matter in the solar system is much less than the mass of the Sun, we can neglect the last term in the denominator of equation (2). Also we assume that the dark matter is non-relativistic, i.e. that $p \ll \rho_{0}$, and accordingly we can neglect the last term in the numerator. Then the TOV equation reduces to

$$
\frac{d p}{d r}=-\left(\rho_{0}+p\right) \frac{M+\frac{4 \pi}{3} \rho_{0} r^{3}}{r(r-2 M)}
$$

Integration leads to

$$
p=\frac{K_{1}}{\sqrt{1-\frac{2 M}{r}}}\left(\frac{r}{2 M}-1\right)^{-16 M^{2} \pi \rho_{0} / 3} \exp \left[-\frac{2 \pi}{3} \rho_{0}\left(4 M r+r^{2}\right)\right]-\rho_{0},
$$

where $K_{1}$ is a constant of integration. With the assumptions that $M^{2} \rho_{0} \ll 1$ and $M \ll r$, this equation simplifies to

$$
p=\frac{K_{1}}{\sqrt{1-\frac{2 M}{r}}} e^{-\frac{2 \pi}{3} \rho_{0} r^{2}}-\rho_{0} .
$$

The constant $K_{1}$ can be determined as follows: let $p_{0}$ be the pressure at $r=r_{\text {surf }}$ where $r_{\text {surf }}$ is the surface of the central mass (the Sun in our case). Within our approximations,

$$
K_{1}=\rho_{0}+p_{0}
$$

The relativistic equation of hydrostatic equilibrium, $T_{; \nu}^{\mu \nu}=0$, where $T^{\mu \nu}$ is the energymomentum tensor of the dark matter, leads to

$$
\frac{d p}{d r}=-\left(\rho_{0}+p\right) \frac{d \mu}{d r}
$$

Integration yields

$$
g_{t t}=-e^{2 \mu}=-K_{2}\left(\frac{\rho_{0}+p_{0}}{\rho_{0}+p}\right)^{2}
$$


where $K_{2}$ is a new integration constant. Inserting the pressure from equations (3) leads to

$$
g_{t t}=-K_{2}\left(1-\frac{2 M}{r}\right) e^{\frac{4 \pi}{3} \rho_{0} r^{2}} .
$$

The constant $K_{2}$ can be determined by demanding that $g_{t t}=-1$ at the surface $r=r_{\text {match }}$ where the pressure equals the galactic dark matter pressure $p_{\mathrm{G}}$. This radius is given by

$$
r_{\text {match }}=\left[\frac{3}{2 \pi \rho_{0}} \ln \left(\frac{\rho_{0}+p_{0}}{\rho_{0}+p_{\mathrm{G}}}\right)\right]^{1 / 2}
$$

and hence

$$
K_{2}=\exp \left(-\frac{4 \pi}{3} \rho_{0} r_{\text {match }}^{2}\right)=\left(\frac{\rho_{0}+p_{\mathrm{G}}}{\rho_{0}+p_{0}}\right)^{2} \approx 1
$$

according to the assumption that $p \ll \rho_{0}$.

Our model of the dark matter filled space-time in the solar system is thus represented by the approximate line-element

$$
d s^{2}=-\left(1-\frac{2 M}{r}+\frac{4 \pi}{3} \rho_{0} r^{2}\right) d t^{2}+\frac{d r^{2}}{1-\frac{2 M}{r}-\frac{8 \pi}{3} \rho_{0} r^{2}}+r^{2} d \Omega^{2}
$$

valid outside the Sun and for $r \ll\left(M / \rho_{0}\right)^{1 / 3}$. It should be noted that we only require the validity of this expression inside the solar system, where $\frac{4 \pi}{3} \rho_{0} r^{2}$ is a small number. At larger scales where the dark matter pressure equals its galactic value, the gravitational field is of course determined by the mass distribution of the galaxy.

\section{PERIHELION PRECESSION}

The Lagrange function for a test particle moving in the $\theta=\pi / 2$ plane in the geometry specified by equation (5), is

$$
2 L=-\left(1-\frac{2 M}{r}+\frac{4 \pi}{3} \rho_{0} r^{2}\right) \dot{t}^{2}+\frac{\dot{r}^{2}}{1-\frac{2 M}{r}-\frac{8 \pi}{3} \rho_{0} r^{2}}+r^{2} \dot{\phi}^{2}
$$

where a dot denotes differentiation with respect to the proper time $\tau$ of the particle (a planet). The momenta $p_{\mu} \equiv \partial L / \partial \dot{x}^{\mu}$ are

$$
\begin{aligned}
& p_{t}=\left(1-\frac{2 M}{r}+\frac{4 \pi}{3} \rho_{0} r^{2}\right) \dot{t}=\text { constant } \\
& p_{r}=\left(1-\frac{2 M}{r}-\frac{8 \pi}{3} \rho_{0} r^{2}\right)^{-1} \dot{r} \\
& p_{\phi}=r^{2} \dot{\phi}=\text { constant. }
\end{aligned}
$$

Using the normalization of the momenta $g_{\mu \nu} p^{\mu} p^{\nu}=-1$, we get

$$
\frac{p_{t}^{2}}{g_{t t}}+\frac{p_{r}^{2}}{g_{r r}}+\frac{p_{\phi}^{2}}{g_{\phi \phi}}=-1
$$


To first order in $M$ and $\rho_{0}$, one finds

$$
\dot{r}^{2}=\left(1-\frac{2 M}{r}-\frac{8 \pi}{3} \rho_{0} r^{2}\right)\left(-1-\frac{p_{\phi}^{2}}{r^{2}}\right)+p_{t}^{2}-4 \pi p_{t}^{2} \rho_{0} r^{2} .
$$

Inserting the new radial variable $u \equiv 1 / r$ and noting from equation (6c) that $\dot{r}=$ $-p_{\phi}(d u / d \phi)$, brings equation (可) into the form of an orbit-equation. Differentiation of the resulting expression, and using that $p_{t} \approx 1$ for planets in the solar system, leads to

$$
\frac{d^{2} u}{d \phi^{2}}+u=\frac{M}{p_{\phi}^{2}}+3 M u^{2}+\frac{4 \pi}{3} \frac{\rho_{0}}{u^{3} p_{\phi}^{2}} .
$$

The planetary orbits are nearly circular, and we can treat the perihelion precession as a perturbation from the circular solution, $u=u_{0}$, where

$$
u_{0}=\frac{M}{P_{\phi}^{2}}+3 M u_{0}^{2}+\frac{4 \pi}{3} \frac{\rho_{0}}{u_{0}^{3} p_{\phi}^{2}} .
$$

Substituting $u_{0}(1+\varepsilon)$ for $u$ into equation (8) with $\varepsilon \ll 1$, and using equation (9) and calculating to first order in $\varepsilon$, we get

$$
\frac{d^{2} \varepsilon}{d \phi^{2}}=\left(6 M u_{0}-1-4 \pi \frac{\rho_{0}}{u_{0}^{4} p_{\phi}^{2}}\right) \varepsilon
$$

The Einstein precession coming from the solar mass $M=M_{\odot}$ is

$$
\Delta \phi_{0}=6 \pi M_{\odot} u_{0}
$$

In addition, there is a dark matter induced precession

$$
\Delta \phi_{\text {dark }}=-4 \pi^{2} \frac{\rho_{0}}{u_{0}^{4} p_{\phi}^{2}}
$$

Equations (10) and (11) imply

$$
\Delta \phi_{\text {dark }}=\frac{2 \pi}{3} \frac{\rho_{0}}{M_{\odot} u_{0}^{5} p_{\phi}^{2}} \Delta \phi_{0} .
$$

Let the observed non-Newtonian perihelion precession be denoted by $\Delta \phi_{\text {obs }}$, and its uncertainty by $\delta \phi_{\text {obs }}$. Since $\Delta \phi_{\text {obs }}=\Delta \phi_{0}$ within the uncertainty, the dark matter perihelion precession and the Einstein term are related by $\left|\Delta \phi_{\text {dark }}\right| \leq\left|\delta \phi_{\text {obs }}\right|$. This, together with equation (12), gives

$$
\rho_{0} \lesssim \frac{3}{2 \pi} M_{\odot} p_{\phi}^{2} u_{0}^{5} \frac{\left|\delta \phi_{\mathrm{obs}}\right|}{\Delta \phi_{0}} .
$$

To lowest order $p_{\phi}^{2}=M / u_{0}$. Thus

$$
\rho_{0} \lesssim \frac{3}{2 \pi} \frac{G M_{\odot}^{2}}{c^{2} r_{0}^{4}} \frac{\left|\delta \phi_{\text {obs }}\right|}{\Delta \phi_{0}}
$$


where we have inserted Newton's constant $G$ and the speed of light $c$ in order to simplify the numerical calculations. The perihelion precession of the asteroid Icarus is known with about $8 \%$ accuracy. Its distance from the Sun is 1.076 A.U. With $M_{\odot}=2 \times 10^{33} \mathrm{~g}$ and $r_{0}=1.076$ A.U. $=1.61 \times 10^{13} \mathrm{~cm}$ we obtain

$$
\rho_{0} \lesssim 1.8 \times 10^{-16} \mathrm{~g} / \mathrm{cm}^{3}
$$

This value is about seven orders of magnitude above the mean galactic mass density, and it shows that measurements of the perihelion precession of the planets do not put strict limits on the density of bound dark matter. Note that with this density, the mass of spherically symmetric bound dark matter of constant density (13) within the orbit of Pluto is less than $2 \times 10^{-5} M_{\odot}$ and thus in agreement with our assumptions. Within Uranus we find a limit of

$$
M_{\text {dark }}\left(r_{\mathrm{U}}\right) \lesssim 2 \times 10^{-6} M_{\odot} \approx 0.6 M_{\oplus}
$$

where $M_{\oplus}$ is the Earth's mass. This bound is of the same size as the bound found by Anderson et al. (1989) by numerically analyzing how the orbit of Uranus would be affected

by dark matter. Recently, the bound obtained by such methods has been strengthened to around $0.2 M_{\oplus}$ (Anderseon et al. 1995), but a similar improvement should also be possible using improved perihelion precession observations.

Let us finally check the assumption that the dark matter has a pressure which is much less than its energy density. The maximum pressure is at the center of the solar system. Now, if we require that the model is valid out to the Oort cloud at several thousand A.U. and that macthing to the galactic dark matter distrubution takes place here, we find that the pressure gradient necessary for hydrostatic equilibrium is very small. Using equation (田) and matching at, say, $r_{\text {match }}=5000$ A.U. gives

$$
\frac{p_{0}-p_{\mathrm{G}}}{p_{\mathrm{G}}} \approx 0.0015
$$

Since $P_{\mathrm{G}} \approx 10^{-7} \rho_{0}$, this result also confirms that $p \ll \rho_{0}$.

\section{DISCUSSION}

Dark matter in the solar system would increase the perihelion precession of the planets. Assuming a very simple model for dark matter having a constant density and being kept in hydrostatic equilibrium by a small but positive pressure, we have used the perihelion precession of Icarus to put a limit of $\rho \lesssim 1.8 \times 10^{-16} \mathrm{~g} / \mathrm{cm}^{3}$ on the density of such dark matter in the solar system. This bound is much larger than the mean galacitic density, but we note that the bound is only weakly dependent on the dark matter distribution function and that some dark matter models may predict a local concentration of dark matter energy.

If dark matter exists in the form of ordinary matter, one would expect that orbital perturbations, especially by Jupiter, effectively would clean the inner parts of the solar system of any weakly interacting dark matter (Anderson et al. 1989). In this case, our bound which was derived from an asteroid would be irrelevant. However, cosmological constraints from inflationary models and cosmic nucleosynthesis, strongly suggest that a large part of 
dark matter is non-baryonic. We do not know what the non-baryonic dark matter is. One possibility (Accetta \& Steinhardt 1991; Steinhardt \& Will 1994) is that the dark matter in part is oscillation energy caused by rapid oscillations of Newton's constant. An effective Brans-Dicke field is a consequence of many unification schemes and also an ingredient of extended inflationary models (La \& Steinhardt 1989). Extended inflation would drive the scalar field away from the minimum of its potential, and the field would then start to oscillate when inflation ends. This possibility only illustrates that dark matter may behave rather differently from normal matter and that no stone should be left unturned in the search for the mass which seems to make up most of the universe we inhabit.

\section{ACKNOWLEDGMENTS}

It is a pleasure to thank Slava G. Turyshev for pointing out some useful references. We are also indebted to an anonymous referee for constructive criticism. 


\section{REFERENCES}

Accetta, F. \& Steinhardt, P. J. 1991, Phys. Rev. Lett., 67, 298.

Anderson, J. D., Lau, E. L., Taylor, A. H., Dicus, D. A., Teplitz, D. C., and Teplitz, V. L. 1989, ApJ, 342, 539.

Anderson, J. D., Lau, E. L., Krisher, T. P. Dicus, D. A., Rosenbaum, D. C., and Teplitz, V. L. 1995, ApJ (in press).

Begeman, K. G., Broeils, A. H., \& Sanders, R. H. 1991, MNRAS, 249, 523.

Bekenstein, J. 1992, in Proceedings of the 6th Marcel Grossmann Meeting on General Relativity, ed. H. Sato \& T. Nakamura (Singapore: World Scientific Publ.), 905

Braginsky, V. B. 1994, Class. Quantum Grav., 11, A1.

Braginsky, V. B., Gurevich, A. V., \& Zybin, K. P. 1992, Phys. Lett. A, 171, 275.

Kent, S. M. 1987, AJ, 93, 816.

Klioner, S. \& Soffel, M. 1993, Phys. Lett. A, 184, 43.

La, D. \& Steinhardt, P. J. 1989, Phys. Rev. Lett. 62, 376.

Lake, G. 1989, ApJ, 345, L17.

Milgrom, M. 1983, ApJ, 270, 365.

Milgrom, M. 1988, ApJ, 333, 689.

Milgrom, M. 1991, ApJ, 367, 490.

Nordtvedt, K. L. 1994, ApJ, 437, 529.

Nordtvedt, K. L., Müller, J. \& Soffel, M. 1995, A\& A, 293, L7.

Sanders, R. H. 1990, Astron. Astrophys. Rev., 2, 1.

Soleng, H. H. 1993, BAAS, 25, 796.

Soleng, H. H. 1994, Gen. Rel. Grav., 26, 149.

Soleng, H. H. 1995, Gen. Rel. Grav., 27, 367.

Steinhardt, P. J. \& Will, C. M. 1995, High-frequency oscillations of Newton's constant induced by Inflation, Phys. Rev. D (in press).

Tremaine, S. 1992, Physics Today, 45, 28.

Tully, R. B. \& Fisher, J. R. 1977, A\&A, 54, 661.

Zhytnikov, V. V. and Nester, J. M. 1994, Phys. Rev. Lett., 73, 2950. 